\title{
Tandem Chromosomal Duplications in Salmonella typhimurium: Fusion of Histidine Genes to Novel Promoters
}

\author{
R. Philit Anderson† and John R. Roth \\ Department of Biology \\ University of Utah \\ Salt Lake City, Utah 84112, U.S.A.
}

(Received 16 August 1977)

\begin{abstract}
Salmonella strains harboring tandem chromosomal duplications have been identified following selection for expression of a histidine biosynthetic gene whose promoter is deleted. In such strains, tandem duplications fuse the selected his gene to "foreign" regulatory elements, thereby allowing gene expression. Selection is made for his $\mathrm{D}^{+}$activity in deletion strain his OG203. Among the revertants, strains harboring tandem chromosomal duplications have been identified by a number of their properties. (1) Their HisD ${ }^{+}$phenotype is genetically unstable. (2) Such instability is dependent on recombination (recA) activity. (3) Genetic tests demonstrate that these strains are merodiploid for large regions (up to $25 \%$ ) of the Salmonella genome. (4) Recipient strains that inherit the HisD ${ }^{+}$phenotype of these duplication-carrying revertants also inherit the donor's merodiploid state. (5) In certain revertants the functional his $\mathrm{D}^{+}$ gene and the sequence which promotes merodiploid transductant formation are linked to chromosomal markers located far from the normal his region.

Previous reports have concluded that the instability of strains isolated by this solcetion is duo to translocation of the $h i s \mathrm{D}^{+}$gone to an extrachromosomal slement (the pi-histidine factor). We believe that in all strains we have tested (33 independent isolates) instability can better be accounted for as due to tandem duplication events which permit expression of his D. At least two mechanisms are responsible for duplication formation. One mechanism is dependent on recombination function and generates identical revertants having a duplication of $16 \%$ of the chromosome. A second mechanism operates independently of recombination activity; individual duplications produced by this process have variable endpoints.
\end{abstract}

\section{Introduction}

Duplication of genetic material has been suggested to be of importance in molecular evolution (Hegeman \& Rosenberg, 1970; Ohno, 1970). Duplications may increase the gene dosage of a required allele, provided fixed heterozygosity of polymorphic variants, or supply the redundant DNAs needed for genetic divergence. Within the past several years methods have been developed for the detection and analysis of tandem genetic duplications in bacteria and their phages. The literature on this work has recently been reviewed (Anderson \& Roth, 1977a). Selections designed to detect cells harboring tandem duplications have generally been based on either the increased gene dosage that the duplication event confers, the heterozygosity that the merodiploid condition allows, or properties of the novel base sequence that is located at the join point between tandemly duplicated regions. This base sequence (often termed the

$\dagger$ Present address: Medical Research Council Laboratory of Molecular Biology, Hills Road. Cambridge, CB2 2QH, Englund. 
novel joint; Hershey, 1970) is not found in the haploid chromosome from which a tandem duplication is derived. Under appropriate conditions, this novel base sequence may confer a selectable phenotype; cells harboring a duplication may then be detected. Detection methods of this type most frequently involve selection for turn-on of genes whose expression has been prevented by either polarity effects or inactivation of promoter elements. Tandem duplications may serve to fuse intact, unexpressed structural genes to functional promoters. Thus, the novel base sequence results from the juxtaposition of a functioning promoter and the structural gene whose expression is selected. This novel sequence is quite analogous to those formed when fusion of operons is achieved by the deletion of intervening material (Miller et al., 1970).

Selections of this general nature have detected in Escherichia coli tandem duplications of the argECBH bipolar operon (Glansdorff \& Sand, 1968; Elseviers et al., 1969,1972; Cunin et al., 1970), of the trp operon (Jackson \& Yanofsky, 1973), of the galETK operon (Hill \& Echols, 1966; Morse, 1967; Ahmed, 1975), of the tryptophanase gene (Yudkin, 1977), of the bacteriophage T4rIIB cistron (Freedman \& Brenner, 1972), and of the bacteriophage P2 early genes $A$ and $B$ (Chattoraj \& Inman, 1974; Bertani \& Bertani, 1974). In each case, tandem duplications cause constitutive expression of the selected gene(s). The amount of duplicated material was generally found to be rather small (1 to 100 genes). Identification of tandem duplications as being responsible for the selected phenotypes has largely been the result of genetic analysis. The most frequently used criteria for identification of tandem duplications are merodiploidy for nearby genetic markers and genetic instability that is dependent on recombination function. In the case of bacteriophage P2, DNA-DNA heteroduplex analysis has provided direct physical evidence for the tandem duplication event (Chattoraj \& Inman, 1974).

Ames et al. (1963) have described a selection in the histidine operon of Salmonella typhimurium very similar to those outlined above. Genetically unstable strains may be obtained by selecting for expression of his gene products whose synthesis has been prevented by deletion of the his operator-promoter region. Deletion hisOG203 removes the his operator-promoter and a portion of the hisG structural gene (see Fig. 1). It leaves the remaining his genes intact but unexpressed. Expression of $\mathrm{HisD}^{+}$activity may be selected as the ability to utilize the intermediate histidinol as a source of histidine. Among the $\mathrm{HisD}^{+}$revertants of his-203 is a class which is highly unstable for its selected phenotype. When such $\mathrm{HisD}^{+}$clones are grown nonselectively, HisD ${ }^{-}$segregants accumulate at a high frequency; these segregants are identical to the parental deletion mutant, his-203. In the original description of this phenomenon (Ames et al., 1963) and in subsequent investigation (Levinthal \& Yeh, 1972), the instability of these strains was interpreted as evidence that the functional

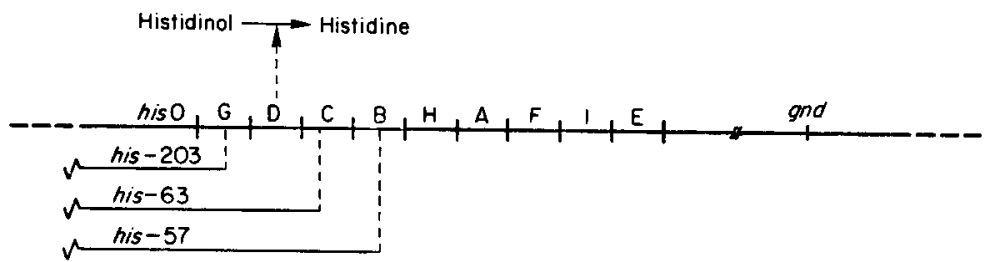

Fig. 1. A genetic map of the histidine operon. Deletion his.203 exhibits no detectable expression of $h i s \mathrm{D}$ and $h i s \mathrm{C}$ enzymes. The remaining genes (his $\mathrm{B}$ through his $\mathrm{E}$ ) are weakly expressed by the low-level constitutive promoter $P 2$, located between his $\mathrm{C}$ and hisB (Atkins \& Loper, 1970). 
his $\mathrm{D}^{+}$gene had been translocated to an extrachromosomal plasmid element (termed the pi-histidine factor). The pi-his factor was considered to replicate autonomously. and instability was thought to result from unequal segregation of this plasmid.

Numerous cases of genetic instability in both Salmonella and E. coli have been attributed to the occurrence of tandem chromosomal duplications in these organisms. It occurred to us that many of the characteristics exhibited by pi-his revertants are alsu exhibited by strains harboring known tandem duplications of the histidine operon. Such duplications have been isolated following generalized transductional crosses that enrich for complementing heterogenotes (Anderson et al., 1976). In this paper we propose that pi-his revertants of deletion his-203 contain tandem chromosomal duplications which fuse the duplicated his $\mathrm{D}$ gene to functional promoter elements. that this structure provides for expression of the his $\mathrm{D}^{+}$gene, and that loss of $\mathrm{His}^{+}$ activity results from homologous recombination beiween the two copies of duplicated material; such recombination events excise intervening material and result in loss of the functional his $\mathrm{D}^{+}$gene.

The genetic characteristics of pi-his revertants (instability, merodiploidy, and transducibility of the merodiploid condition) are standard behavior of tandem chromosomal duplications. Two classes of pi-his duplications have been found. One class is formed by a recombination-dependent process; independent isolates of this type are duplicated for an identical $16 \%$ of the Salmonella chromosome. A second, heterogeneous class is formed by a recombination-independent mechanism; individual isolates are duplicated for various amounts of nearby material.

A preliminary account of this work has been presented elsewhere (Anderson \& Roth, $1977 b)$.

\section{Materials and Methods}

(a) Media and grouth conditions

Vogel \& Bonner (1956) E medium containing $2 \%$ glucose was used as minimal medium. When required, this medium was supplemented with $0.1 \mathrm{~mm}$ (excess) or $0.005 \mathrm{~mm}$ - (lim. iting) histidine, $2.0 \mathrm{~mm}$-histidinol, $0.4 \mathrm{~mm}$ each of adenine, guanine and arginine, $0.05 \mathrm{~mm}$ thiamin, and approx. $0 \cdot 3 \mathrm{~mm}$-other amino acids. DL amino acids were often used, but the concentrations given are for the $L$ isomer. 3-Amino-1,2,4-triazole (Aldrich) was added at $20 \mathrm{~mm}$ final concn. When desired as a sole carbon source, D-sorbitol (Sigma) was added at $0 \cdot 2 \%$ to $\mathrm{E}$ medium from which glucose and citrate had been omitted. Difco nutrient broth $(0 \cdot 8 \%)$ containing $0.5 \% \mathrm{NaCl}$ was used as complex medium. Solid media contained $1.5 \%$ Difco agar. All incubations were at $37^{\circ} \mathrm{C}$. Liquid cultures were aerated by gyrotory shaking.

\section{(b) Bacterial strains}

The genotypes and sources of selected strains used in this study are shown in Table 1. Strains with TT designations are those in our collection that either contain or are descended from a strain containing a copy of the translocatable tetracycline-resistance determinant $\operatorname{Tn} 10$ (Kleckner et al., 1975). All strains are derivatives of $S$. typhimurium strain LT2.

Strain TK4178 (his-203 $\mathrm{srl}$-201) was isolated following diethyl sulfate mutagenesis of his-203 and 2 cycles of penicillin enrichment for $\mathrm{Srl}^{-}$(sorbitol non-utilizing) clones. Both mutagenesis and pencillin enrichment were performed according to the procedures of Roth (1970). The mutation srl-201 is approx. 50\% linked to recAl by P22mediated generalized transduction. The close proximity of these two loci in $E$. coli has been described (McEntee, 1976; A. J. Clark, personal communication). Strain TR4192 (his-203 srl-201 recAl strA) was constructed by mating strain TR4178 with donor strain TR2246 (metA22 recAl strA HfrB2). Streptomycin-resistant conjugants were selected and the desired $\mathrm{srl}^{-} \mathrm{recA} \mathrm{A}^{-}$recombinant was identified among the progeny. Strain TR295I (his-63 
TABLe 1

List of strains

\begin{tabular}{|c|c|c|}
\hline Strain & Genotype & Source \\
\hline$h i s-203$ & hisOG203 & P. E. Hartman \\
\hline his-63 & $h i s \mathrm{OGDC63}$ & P. E. Hartman \\
\hline TR2246 & met $\mathrm{A} 22 \operatorname{rec} \mathrm{Al} s t r \mathrm{~A} \quad \mathrm{Hfr} \mathrm{B} 2$ & J. Wyche \\
\hline TR295I & $h i s-63$ rec $\mathrm{Al} s t r \mathrm{~A}$ & TR2246 $\times$ his. 63 \\
\hline TR4178 & $h i s-203$ srl-201 & This paper \\
\hline TR4192 & his-203 srl-20l recAl strA & TR2246 $\times$ TR4178 \\
\hline PS29 & his-57 $(p i-2)$ & C. Gritzmacher \\
\hline TT14 & $\operatorname{met} \mathrm{C} 1975: \operatorname{Tn} 10$ & This paper \\
\hline TT126 & 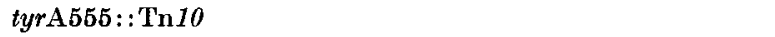 & This paper \\
\hline TT142 & 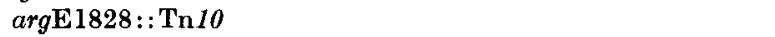 & This paper \\
\hline TT146 & $\arg \mathrm{B} 1832: \operatorname{Tn} 10$ & This paper \\
\hline TT169 & 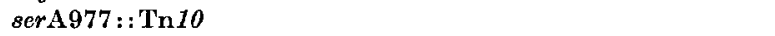 & This paper \\
\hline TT172 & cysG1510::Tn10 & This paper \\
\hline TT173 & cys $\mathrm{C} 1511: \operatorname{Tn} 10$ & This paper \\
\hline TT215 & lysA565: $: \operatorname{Tn} 10$ & This paper \\
\hline TT233 & $m e t \mathrm{~F} 877:: \operatorname{Tn} 10$ & This paper \\
\hline TT278 & guaA554:: $\operatorname{Tn} 10$ & This paper \\
\hline TT287 & purC882: :Tn10 & This paper \\
\hline TT315 & purG $1739:: \operatorname{Tn} 10$ & This paper \\
\hline TT317 & purF $1741:: \operatorname{Tn} 10$ & This paper \\
\hline TT418 & $g l y \mathrm{~A} 540:: \operatorname{Tn} 10$ & This paper \\
\hline TT744 & his $-63 \arg \mathrm{B} 1832:: \operatorname{Tn} 10$ & TT146 $\times$ his 63 \\
\hline TT1720 & aroD5 $z h f-105:: \operatorname{Tn} 10$ & G. Ames \\
\hline TT1738 & aroD5 hisW1824 metG319 purF145 strA his-63 zgf-2::Tn10 & This paper \\
\hline NK 186 & cysA $1539:: \operatorname{Tn} 10$ & N. Kleckner \\
\hline
\end{tabular}

All strains are derivatives of $S$. typhimurium strain LT2. See Materials and Methods for the derivation of strains original to this paper.

recAl $s t r \mathrm{~A}$ ) was constructed by a similar cross using $h i s-63$ as the $\mathrm{F}^{-}$recipient. Recombination deficiency $\left(\mathrm{recA}^{-}\right)$was scored as sensitivity to approximately $400 \mathrm{ergs} / \mathrm{mm}^{2}$ of ultraviolet light irradiation. Strain PS29 (his-57 [pi-2]) harbors the reversion event pi-2 originally described by Ames et al. (1963). PS29 was used as our source of $p i$-2 and was kindly supplied by C. Gritzmacher.

A large number of nutritional auxotrophs resulting from insertion of the $\operatorname{Tn} 10$ element (Kleckner et al., 1975) have been isolated in our laboratory as a co-operative effort. The sites of insertion have been identified in many of these auxotrophs by as many as 3 independent tests: (1) the ability of selected biosynthetic intermediates to fulfil nutritional requirements (crystal tests); (2) a demonstration of transductional linkage of Tn10 insertions to known genetic markers; and (3) the marked reduction in numbers of prototrophic recombinants obtained when Tn10 insertions are crossed with allelic mutations of known genotype. The results of these tests have led to the unambiguous assignment of many Tn10 insertions to defined genes. Identification of the insertion site in TT317 (purF1741:: Tn10) was made by J. Gots (personal communication).

Strain TT1738 (aroD5 hisW1824 metG319 purF145 strA his-63 zgf-2::Tn10) was derived from strain SB562 (aroD5 hisW1824 metG319 purF145 strA [P22]) in a manner designed to eliminate the P22 prophage present in SB562. Phage grown on TT 184 (proA622::Tn10) was used to transduce SB562 selecting tetracycline resistance. Since proAB and ataA (the P22 prophage attachment site) are transductionally linked, many of the $\mathrm{Tet}^{\mathrm{R}} \mathrm{Pro}^{-}$recombinants had rocombined out the prophage. One recombinant of this type (TT1723) was transduced to $\mathrm{Pro}^{+}$with phage grown on LT2. The resulting Pro ${ }^{+}$Tet $^{\mathrm{S}}$ recombinant (TT1727) was then used as a recipient for donor strain TT1721 (his-63 zgf-2::Tn10). This donor contains 
a Tn10 insertion (isolated by F. Chumley) in "silent" DNA approx. 90\% linked to the delelion his-83. Using this donor, most Tet ${ }^{\mathbb{R}}$ transductants inherited his.63 in addition to the Tn10 element. One such recombinant is strain TT1738.

\section{(i) Isolation of pi-his revertants}

\section{(c) Genetic techniques}

In order to measure the frequency of $\mathrm{HisD}^{+}$reversion of selected strains, nutrient broth grown cells were collected by centrifugation, washed with a minimal salts solution, and concentrated 10-fold. Samples were spread on E minimal plus $2.0 \mathrm{~mm}$-histidinol plates. After 3 days incubation, the numbers of $\mathrm{HisD}^{+}$colonies were scored. For the isolation and analysis of independent pi-his revertants, individual nutrient broth cultures were inveulated with single colonies of either strain TR4178 (his-203 srl-201) or TR4192 (his-203 srl.201 recAl strA). Following overnight growth, portions were spread individually onto E minimal plus 2.0 mm-histidinol plates. From cach culture, I revertant was picked, purified, and analyzed further. Unstable isolates were assigned pi-his allele numbers; $p i-401$ through $p i-421$ were isolated from TR4178 (rec $\left.\mathrm{A}^{+}\right) ; p i-422$ through $p i-432$ were isolated from TR4192 (rect- $A^{-}$.

\section{(ii) Visualization of $\mathrm{HisD}^{-}$segregants}

In order to test the genetic stability of the HisD ${ }^{+}$phenotype of various strains, single colonies of these strains were picked from selective plates ( $\mathbf{E}$ minimal plus histidinol) and allowed to grow for 15 to 20 generations non-selectively in liquid nutrient broth. These cultures were then diluted and single colonies were spread on minimal plates containing both histidinol $(2 \mathrm{~mm})$ and a limiting concentration of histidine $(0.005 \mathrm{~mm})$. Under these conditions, HisD ${ }^{+}$clones form large round colonies, while $\mathrm{HisD}^{-}$clones form small, flat. easily distinguishable colonies.

\section{(iii) Transductions}

$\Lambda$ derivative of the high-transducing phage P22 HT105/1 (Schmieger, 1971) was used in all transductions. This derivative (P22 HT105/1 int-201) was obtained in our laboratory by $\mathrm{G}$. Roberts following hydroxylamine mutagenesis of P22 HT105/1 and a sereen for non-lysogenizing variants. For transductions, plates were spread with a mixture of $2 \times 10^{8}$ to $6 \times 10^{8}$ recipient cells and approx. $10^{8}$ phage particles. Transductant clones were scored after 2 to 4 days incubation at $37^{\circ} \mathrm{C}$. When transductants were to be used in further work, clones were purified 3 times selectively, verified to be free of phage, and preserved.

\section{(iv) Preservation of unstable strains}

Cultures of unstable duplication strains in liquid medium were supplemented to contain $8 \%$ dimethyl sulfoxide and frozen at $-70^{\circ} \mathrm{C}$. We have found this technique exceptionally good for long-term preservation of strains whose desired genetic determinants are unstable (duplications, $\mathrm{F}^{\prime}$ episones, Hfr strains, etc.).

\section{Results}

(a) The genetic consequences of tandem duplications

Before presenting specific models or experimental details, it is important to first consider several formal genetic aspects of tandem duplications.

\section{(i) Tandem duplications generally cause no loss of function}

The reasons why tandem duplications are generally non-destructive can be seen in the diagram of a tandem duplication presented in Figure 2. In the chromosome carrying the duplication, the only impropriety in base sequence is located at the marked point between the two tandemly repeated copies $(i-d)$. This impropriety does 


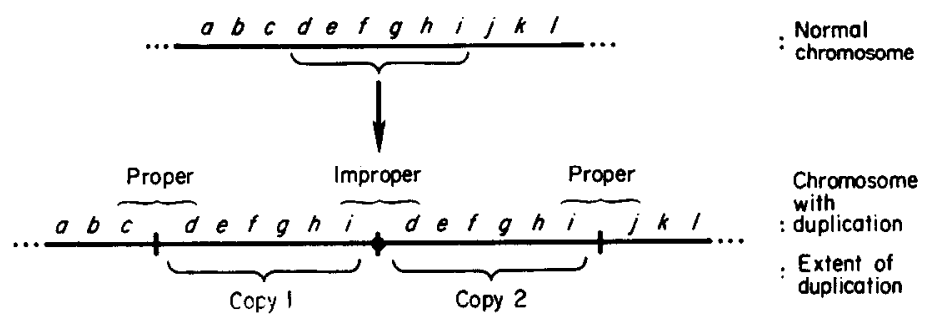

Fia. 2. Tandem duplication of the chromosomal segment defghi. Lower-case letters are nongonetic indications of hypothotical base sequences.

not lead to a loss of function, because proper versions of these sequences are present elsewhere ( $c-d$, at the left; $i$ - $j$, at the right). Thus, large tandem duplications do not destroy any genetic information. Exceptions to this rule are encountered when both endpoints of a duplication are located within a single gene or operon.

(ii) Tandem duplications may be of unlimited size.

Since duplications lead to no loss of function, they are unlikely to be deleterious. Thus, even very large duplications may be maintained. The only restriction on the permissible size of a duplication might be the ability of the cell to replicate and segregate this large chromosome faithfully.

(iii) Tandem duplications are subject to frequent loss

Tandem duplications are unstable genetic structures. Reciprocal recombination between the two copies of duplicated material serves to excise intervening DNA and yields haploid chromosomes. Since this process involves legitimate recombination between homologous sequences, it might be expected to occur frequently. Loss of the duplication should depend strongly on recombination.

\section{(iv) Extremely large tandem duplications can be transduced}

As mentioned earlier, the only novel base sequence in chromosomes carrying a tandem duplication is located at the join point between copies of the duplicated region. At this point, sequences are made contiguous that would be widely separated in a normal chromosome. Transduction of this join point into a normal (haploid) recipient can serve to re-establish the donor's duplication state in recipient cells. This is possible even when the region included in the duplication is much too large to be carried by a single transducing fragment. Recombination events which account for this behavior are depicted in Figure 3. A transducing fragment that carries the join point of a tandem duplication contains base sequence homology to two widely separated regions on recipient chromosomes. When such a fragment enters recipient cells, reciprocal recombination events between the fragment and two recipient chromosomes regenerate the donor's duplication state. In the resulting recombinant, most of the duplicated material is derived from the recipient chromosome; only material immediately adjacent to the join point is derived from the donor. Thus, transduction of large tandem duplications may be detected, provided the selected donor marker and the join point between duplicated material are cotransducible. Transductional events such as those described in Figure 3 were first suggested by 

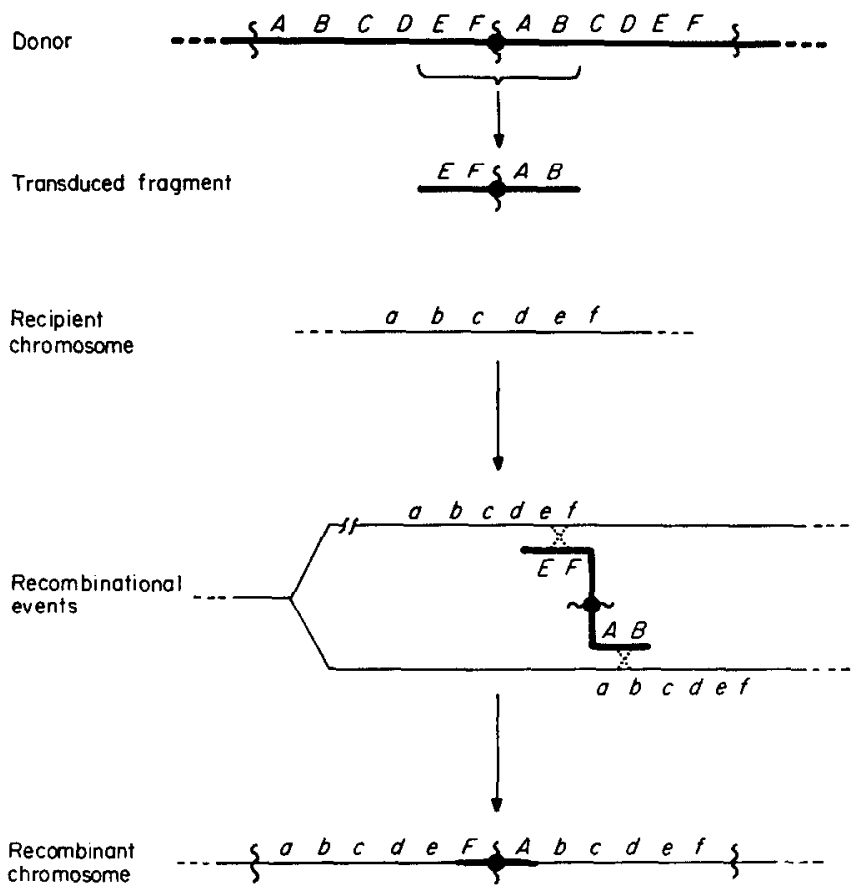

FIa. 3. A mechanism for transduction of large tandem duplications (Campbell, 1965; Hill et al., 1969). Recipient and donor DNA are light and bold lined, respectively. Dotted lines represent reciprocal recombination events.

Campbell (1965). Strong genetic evidence in support of these events was subsequently presented (Hill et al., 1969).

(b) Isolation and behavior of pi-his revertants

Deletion his0G203 removes the operator-promoter and a portion of the first structural gene of the histidine operon (see Fig. 1). The his D gene in strain his-203 remains structurally intact, but unexpressed. Three classes of $\mathrm{HisD}^{+}$(histidinol utilizing) revertants of his-203 have been described (Ames et al., 1963; St. Pierre, 1968): (1) deletion mutations that extend the deleted region and fuse the his $\mathrm{D}$ gene to nearby constitutive promoters; (2) point mutations within the residual hisG gene that provide a new promoter for hisD expression; and (3) unstable mutations that "revert" at a high frequency to their parental genotype. Such unstable mutations have been designated pi-his revertants, due to the presumed translocation of the functioning $h i s$ genes to an extrachromosomal element (the $p i$-his factor).

In the original description of this phenomenon, Ames et al. (1963) demonstrated that: (1) His $\mathrm{D}^{-}$segregants that arise from unstable pi-his revertants are identical to the parental deletion $h i s-203$; (2) recipient strains that have inherited the $\mathrm{His}^{+}$ phenotype of $p i$-his revertants are themselves genetically unstable; (3) all his genes (except $h i s \mathrm{G}$ ) are constitutively expressed in pi-his revertants; and (4) the functional $h i s \mathrm{D}^{+}$gene in pi-his revertants is not transductionally linked to the normal his operon.

We propose a model for the structure of pi-his revertants based on tandem duplication of chromosomal material, 
(c) A proposed structure for pi-his revertants

Our model to account for the structure of pi-his revertants is shown in Figure 4. There are promoters (termed $P^{\prime}$ ) located at some distance from the histidine operon which have the same direction of transcription as does the histidine operon. Tandem duplication of material from a point within the his operon to a point within the transcription unit $P^{\prime}$ leads to fusion of the duplicated histidine genes to these "foreign" regulatory elements. In the resulting structure, the duplicated his $\mathrm{D}^{+}$gene is expressed under control of the promoter $P^{\prime}$, replicated as a part of the chromosome, lost as a result of recombination between copies of duplicated material, and transduced by the mechanism outlined in Figure 3 (see above). The non-destructive nature of tandem duplications suggests that the amount of duplicated material might be quite large. In the following two sections we shall present evidence which confirms important predictions of this model. In later sections the mechanisms of duplication will be considered. We should like to distinguish between two types of duplications: those formed by recombination-dependent and recombination-independent processes.

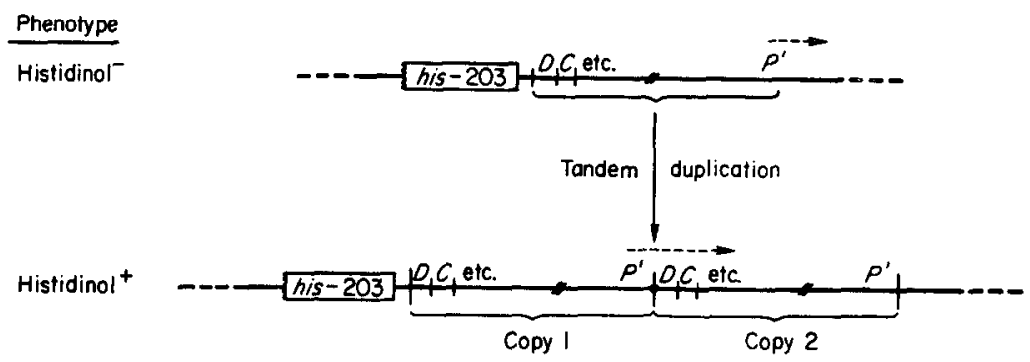

FIG. 4. A proposed structure for pi-his revertants. The broken arrows indicate the direction of transcription from promoter $P^{\prime}$.

\section{(d) Instability of pi-his revertants is dependent on recombination}

Tandem genetic duplications generate haploid segregants as a result of homologous recombination between duplicated regions (Campbell, 1963). Thus, if the instability of pi-his is due to a tandem duplication, this instability should be evident only in recombination-proficient backgrounds. The crosses shown in Figure 5 demonstrate that this is true for pi-his revertants. $\operatorname{rec} \mathrm{A}^{-}$alleles were introduced into pi-his revertants by use of the transductionally linked marker $\mathrm{srl}^{-}$. $\mathrm{Srl}^{-}$strains are unable to utilize D-sorbitol as a sole carbon source. In the first cross shown in Figure 5, a $s r l^{+} r e c \mathrm{~A}^{-}$donor has been used to transduce a number of independent $s r l^{-} r e c \mathrm{~A}^{+}$pi-his revertants, and the isogenic $\operatorname{Rec}^{+}$and $\operatorname{Rec}^{-}$recombinants were identified among the progeny. $\mathrm{Rec}^{+}$recombinants exhibit the same high frequency of $\mathrm{HisD}^{-}$segregants characteristic of pi-his revertants (average $=35 \%$ segregants following 15 to 20 generations of non-selective growth for the six strains tested). Rec ${ }^{-}$recombinants exhibit no $\mathrm{HisD}^{-}$segregants $(<0.02 \%)$. Yet, these stable strains still contain the $p i$-his structure; when they are used as donors in crosses designed to recover pi-his from them (cross no. 2), the characteristic instability reappears in the resulting rec $\mathrm{A}^{+}$pi-his recombinants. Stability of $p i$-his in recA- backgrounds has been demonstrated for five independent $p i$-his revertants isolated by us $(p i-404,-413,-414,-420$, and -421 ) and for the revertant pi-2 described by Ames et al. (1963). Based on these 

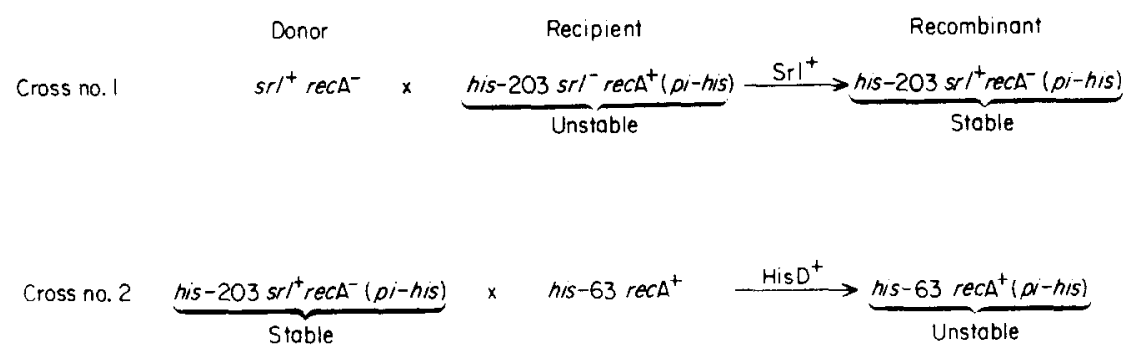

Fia. 5. Transductional crosses demonstrating that instability of pi-his rovertants is dopendent on recombination. Selected phenotypes are indicated above the arrows. Multiply marked strains used were TR2951 (his-63 srl ${ }^{+}$recAl strA) and 6 independent pi-his revertants (pi-404, pi-413, $p i-414, p i-420, p i-421$ and $p i-2$ ). The revertant $p i-2$ is described by Ames et al. (1963). Mutation his-63 is a large deletion whose map position is depicted in Fig. 1. All crosses were performed as described in Materials and Methods.

experiments, we conclude that $p i$-his revertants are at least 1700 -fold more stable in recombination-deficient backgrounds.

\section{(e) pi-his revertants are merodiploid for large chromosomal regions}

By a variety of genetic techniques, we have determined the amount of duplicated material in 33 independent $p i$-his revertants. Our method of isolating $p i$-his revertants guarantees them to be of independent origin (see Materials and Methods). Of these revertants, 22 were isolated in a rec $\mathrm{A}^{+}$genetic background; the merodiploid content of these strains is presented in this section. The remainder were isolated in a rec $\mathrm{A}$ background; the results of these tests are presented in a later section (see below) that describes the role of recombination in $p i$-his formation.

The basic scheme for determining the extent of duplicated material in pi-his revertants has been to determine whether they can be made hetcrozygous for nearby genetic markers. If they can, then these strains must be merodiploid for those markers. Three different techniques have been used to determine the merodiploidy of nearby markers. In most cases, merodiploidy has been tested through the use of auxotrophic mutations generated by insertion of the transposable tetracycline-resistance determinant $\operatorname{Tn} 10$ (Kleckner et al., 1975). These auxotrophs result from the linear insertion of the $\operatorname{Tn} 10$ element into defined structural genes. The $\operatorname{Tn} 10$ element specifies a selectable phenotype (tetracycline resistance), as well as causing the auxotrophy phenulype. Selection for inheribance of Tn10 (by selecting tetracycline resistance) demands that recipients also inherit the lesion caused by $\operatorname{Tn} 10$ insertion. When haploid strains inherit such Tn10 insertions, they inherit the donor's auxotrophy as well. However, if the recipient strain is merodiploid for the $\operatorname{Tn} 10$ insertion site, tetracycline-resistant recombinants remain prototrophic, due to the presence of a second (wild-type) copy of the gene involved and the recessive nature of these mutations. Transductional crosses of this type allow rapid testing of whether a given strain is merodiploid for known $\operatorname{Tn} 10$ insertion sites. When $\operatorname{Tn} 10$ insertions were not available for markers to be tested, alternative procedures were used. For testing of met $\mathrm{G}$ and $s r l$, pi-his revertants were either isolated in or transduced into genetically marked backgrounds ( $m e t \mathrm{G}^{-}$or $s r l^{-}$). If such strains were merodiploid for met $\mathrm{G}^{-}$or $s r l^{-}$. subsequent transductions (to $\mathrm{MetG}^{+}$or $\mathrm{Srl}^{+}$) yielded clones that were heterozygous. Analysis of the genotypes of the his $\mathrm{D}^{-}$haploid segregant population from these clones 
then revealed any heterozygosity. That is, if both met $\mathrm{G}^{+}$and $m e t \mathrm{G}^{-}$(or $\mathrm{srl}^{+}$and $s r l^{-}$) clones were present among the HisD ${ }^{-}$segregants, the strain from which these segregants arose must have been heterozygous for the met $\mathrm{G}$ (or $s r l$ ) region. Merodiploidy for $r e c \mathrm{~A}$ was similarly tested by transducing $r e c \mathrm{~A}^{-}$alleles into pi-his revertants using the linked marker $\mathrm{srl}^{-}$. These heterozygotes were then further analyzed by screening the $\mathrm{His}^{-}$segregants for the $\operatorname{rec\mathrm {A}^{-}}$ phenotype. $\left(\operatorname{rec} \mathrm{A}^{+} / \mathrm{rec} \mathrm{A}^{-}\right.$ heterozygotes are phenotypically $\mathrm{Rec}^{+}$and therefore unstable.) Merodiploidy of aroD was tested by use of strain TT1720 (aroD5 $z h f-105:$ : Tn 10). This strain harbors a Tn10 insertion (isolated by G. Ames) approximately $40 \%$ linked to the mutation aroD5. When haploid recipients are transduced with this donor, $40 \%$ of Tet $^{R}$ recombinants inherit aroD5. When recipients merodiploid for the aro $\mathrm{D}^{+}$gene are used, no apparent linkage is observed (aroD5 is recessive).

In Table 2 the results of these tests are presented for pi-his revertants that formed in a rec $\mathrm{A}^{+}$background. Among 22 independent $p i$-his revertants investigated, three classes are revealed. Class $\mathrm{I}$ isolates are merodiploid for the nearby locus met $\mathrm{G}$. They are not merodiploid for the next tested marker, purF. Thus, pi-his revertants of this type are merodiploid for a chromosomal segment that is $3 \%$ to $8 \%$ of the Salmonella genome. Class I isolates total 6 of the 22 independent revertants and include the isolate $p i-2$ described by Ames et al. (1963). Class II revertants form the majority (15 of 22 isolates). They are merodiploid for all loci tested (as many as 12) in the region from his through $\arg \mathrm{B}$. Thus, class II revertants harbor a merodiploid region of approximately $16 \%$ of the genome. A single revertant (class III) is merodiploid for each of 17 loci tested in the region from his through $\arg \mathrm{E}$; this strain is merodiploid for approximately $25 \%$ of the Salmonella genome.

It is very important to note that the results presented in Table 2 were obtained with strains that had inherited by transduction the His $\mathrm{D}^{+}$phenotype of the original pi-his revertants. Thus, transduction of the His $\mathrm{D}^{+}$phenotype into new recipients establishes a merodiploid state in these pi-his recombinants. This merodiploid state may even be as large as $25 \%$ of the genome. Considering the transducible nature of large tandem genetic duplications (Hill et al., 1969; see Fig. 3), we interpret these results as evidence that the functional $h i s \mathrm{D}^{+}$gene in $p i$-his revertants is located near the join point of a tandem chromosomal duplication.

For pi-his revertants of the class II type, the location of the duplication join point and the linkage of a functional his $\mathrm{D}^{+}$gene to this join point may be demonstrated quite dramatically. Class II revertants are merodiploid for all loci tested in the region from $h i s$ through $\arg \mathrm{B}$ (see Table 2). They are not merodiploid for the gene lys A. $\arg \mathrm{B}$ and $l y s \mathrm{~A}$ are approximately one minute separated on the Salmonella genetic map. The functional his $\mathrm{D}^{+}$gene in class II revertants may be shown to be linked to the $\arg \mathrm{B}$ gene by the following experiment: when any class II revertant is used as a transductional donor and an $\arg \mathrm{B}^{-} h i s \mathrm{D}^{-}$strain is used as a recipient, approximately $33 \%$ of $\mathrm{Arg}^{+}$transductants inherit the $\mathrm{HisD}^{+}$phenotype non-selectively. These data are shown in Table 3. The resulting $\mathrm{Arg}^{+} \mathrm{HisD}^{+}$recombinants are unstable for both their $\mathrm{Arg}^{+}$and their HisD phenotypes. Moreover, they are merodiploid for the entire chromosomal region from his through $\arg \mathrm{B}$. We presume that these transductants arise as diagrammed in Figure 3 and interpret these results as indicating cotransduction between the $\arg \mathrm{B}$ gene, the join point of a tandem duplication, and a functional $h i s \mathrm{D}^{+}$gene in class II pi-his revertants. Linkage data of this type are strong evidence for a tandem chromosomal duplication contained by pi-his revertants. 


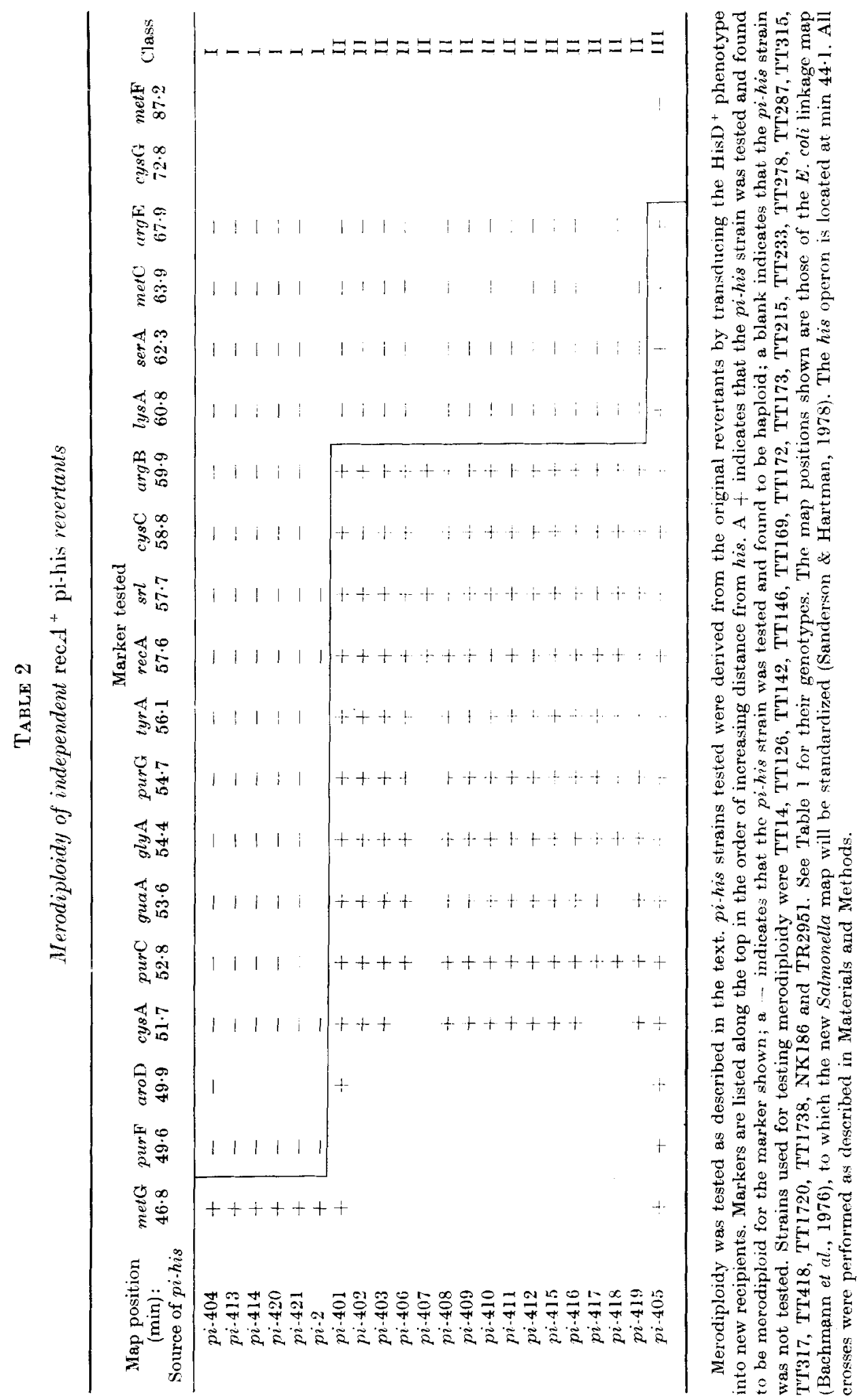


TABLE 3

Cotransduction of $\mathrm{His}^{+}$with $\arg B$ in class $I I$ pi-his revertants

\begin{tabular}{|c|c|c|c|c|}
\hline $\begin{array}{l}\text { Soureo of } \\
\text { donor pi-his }\end{array}$ & $\begin{array}{l}\text { Revortant } \\
\text { class }\end{array}$ & $\begin{array}{l}\Delta r g{ }^{+} \mathrm{HisD}^{-} \\
\text {recombinants }\end{array}$ & $\begin{array}{l}\mathrm{Arg}^{+} \mathrm{HisD}^{+} \\
\text {recombinants }\end{array}$ & $\%$ Cotransduction \\
\hline$p i-401$ & II & 323 & 97 & 23 \\
\hline$p i-402$ & II & 67 & 33 & 33 \\
\hline$p i-403$ & II & 33 & 17 & 34 \\
\hline$p i-406$ & II & 37 & 22 & 37 \\
\hline$p i-407$ & II & 74 & 38 & 34 \\
\hline$p i-408$ & II & 75 & 25 & 25 \\
\hline$p i-409$ & II & 66 & 34 & 34 \\
\hline$p_{i-410}$ & II & 81 & 39 & 33 \\
\hline$p i-411$ & II & 38 & 22 & 37 \\
\hline$p i-412$ & II & 73 & 38 & 34 \\
\hline$p i-415$ & II & 30 & 14 & 32 \\
\hline$p i-416$ & II & 43 & 17 & 28 \\
\hline$p i-417$ & II & 40 & 22 & 35 \\
\hline pi-418 & II & 66 & 34 & 34 \\
\hline$p i-419$ & II & 62 & 38 & 38 \\
\hline$p i-404$ & I & 100 & 0 & $<1$ \\
\hline$p i-405$ & III & 100 & 0 & $<1$ \\
\hline LT2 & $\ldots$ & 100 & 0 & $<1$ \\
\hline
\end{tabular}

The recipient strain is TT744 (his-63 $\operatorname{argB1832::Tn10).~}$

$\mathrm{Arg}^{+}$recombinants were selected on minimal medium plus histidine plates. Transductants were picked and subsequently scored for their HisD phenotype. In each case one representative $\mathrm{Arg}^{+} \mathrm{HisD}^{+}$recombinant was purified and verified to be unstable for both $\mathrm{Arg}^{+}$and $\mathrm{HisD}^{+}$. All crosses were performed as described in Materials and Methods.

\section{(f) Frequency of pi-his reversion}

$\mathrm{HisD}^{+}$revertants of strain TR4178 (his-203 srl-201) are obtained spontaneously at a frequency of $1.7 \times 10^{-9}$ per cell. Of 148 independent revertants, 29 were found to be genetically unstable and therefore classified as $p i$-his revertants. Thus, $p i$-his reversion occurs at a frequency of $3 \times 10^{-10}$ per cell. The nature of the stable revertants has been described (Ames et al., 1963; St. Pierre, 1968). HisD ${ }^{+}$revertants of strain TR4192 (his-203 srl-201 recAl strA) are obtained spontaneously at a frequency of $1.2 \times 10^{-9}$ per cell. As expected, all revertants obtained in the rec $\mathrm{A}^{-}$background are stably $\mathrm{HisD}^{+}$. However, when $\mathrm{rec \textrm {A } ^ { + }}$ alleles are introduced into these revertant strains (using the linked $s r l$ mutation), approximately $5 \%$ (11 of 244 independent revertants) become unstable. Thus, in a recombination-deficient background, pi-his revertants are obtained at a frequency of $5 \times 10^{-11}$ per cell; this figure is approximately sixfold less than in a $r e c \mathrm{~A}^{+}$background. Since instability is only evident after introduction of the $r e c \mathrm{~A}^{+}$allele, these results confirm the observation that recombination is required for pi-his segregation. The sixfold reduced pi-his reversion frequency in rec $\mathrm{A}^{-}$backgrounds appears to be explained by the absence of isolates having their duplication endpoints in the $\arg$-BlysA region. Such isolates are frequent among revertants in a $r e c \mathrm{~A}^{+}$background and are absent among rec $\mathrm{A}$ isolates. Therefore, class II isolates appear to be formed by a recombination-dependent mechanism. This conclusion is based on a comparison of the extent of merodiploidy harbored by pi-his revertants obtained in $r e c \mathrm{~A}^{+}$and $r e c \mathrm{~A}^{-}$backgrounds. These data are presented in the following section. 


\section{(g) Distribution of pi-his duplication endpoints formed in rec $A^{-}$backgrounds}

The extent of the merodiploid region harbored by each of 11 independent pi-his revertants isolated in a rec $\mathrm{A}^{-}$background has been determined. $r e c \mathrm{~A}^{+}$alleles were transduced into 244 independent $\mathrm{HisD}^{+}$revertants of strain TR4192 (his-203 srl-201 recAl str $\mathrm{A}$ ), and 11 unstable pi-his isolates were identified. The $\mathrm{HisD}^{+}$phenotype of these strains was then transduced into new recipients $($ his-63); the resulting $p i$-his recombinants were used to determine the extent of merodiploidy of each isolate. The methods used for detecting merodiploidy were those described in section (e) above. The results of these tests are presented in Table 4 . Among the 11 revertants, seven classes are present. The class I revertant is not merodiploid for metG, and thus contains no detectable merodiploidy. It likely harbors a short duplication whose endpoint is located between his and metG. Class II revertants are distinguished from class III revertants (both of which have endpoints in the metG-purF region), by the fact that the join point between duplicated regions and the functional his $\mathrm{D}^{+}$gene in class II revertants are approximately $10 \%$ linked to metG319. Class III revertants do not exhibit such linkage. Classes IV to VII are each merodiploid to a different extent. 'I'he largest duplication (contained by $p i-431$ ) is approximately $12 \%$ of the genome. The functional his $\mathrm{D}^{+}$gene of each class IV isolate is approximately $4 \%$ linked to the mutation aroD5. Similarly, the his $\mathrm{D}^{+}$gene of $p i-429$ (class VI) is $12 \%$ linked to purG1739:: Tn10.

Conspicuously absent from the pi-his isolates formed in rec $\mathrm{A}^{-}$cells are those having endpoints in the $\arg \mathrm{B}-l y s \mathrm{~A}$ region (class II rec $\mathrm{A}^{+}$revertants). Thus, revertants of this type (a majority of isolates formed in $r e c A^{+}$cells) appear to be generated by a recombination-dependent process. The $11 \mathrm{rec} \mathrm{A}^{-}$revertants are heterogeneous with respect to the size of the merodiploid region. However, identical independent isolates are occasionally obtained. This contrasts sharply with the results obtained in a rec. $\mathrm{A}^{+}$ background. In that case, most of the isolates (15 of 22 ) have the same $16 \%$ of the chromosome duplicated. The remainder arise at a frequency comparable to those obtained in the rec $\mathrm{A}^{-}$background by recombination-independent mechanisms.

\section{(h) Duplicated material within the his operon}

By testing each pi-his revertant for merodiploidy of a series of nearby loci, it was possible to determine the position of one endpoint of the duplicated material (see alsove). The highly non-random distribution of these endpuints (in rec $\mathrm{A}^{+}$isolates) prompted us to map the location of the second endpoint, located near the his $\mathrm{D}$ gene. We sought to determine whether these endpoints exhibit a non-random distribution as well. Ames et al. (1963) demonstrated that pi-his revertants complement all his mutations except those in hisG. Levinthal \& Yeh (1972) presented evidence that the breakpoint within the operon for expression of his $\mathrm{D}$ is located at a unique site within the hisG gene. We have determined precisely the location within the his operon of the duplication endpoint in each of our pi-his revertants (both rec $\mathrm{A}^{+}$and $r e c \mathrm{~A}^{-}$ isolates). This task was made easier by the availability of a revised genetic map of the his G gene (Hoppe et al., manuscript in preparation). A large number of deletions affecting $h i s \mathrm{G}$ has recently become available (Scott et al., 1975; Ino et al., 1975). These mutations have allowed very sensitive fine-structure mapping of the gene. The resolution of the new map is quite high, due to the use of a high-frequency generalized 


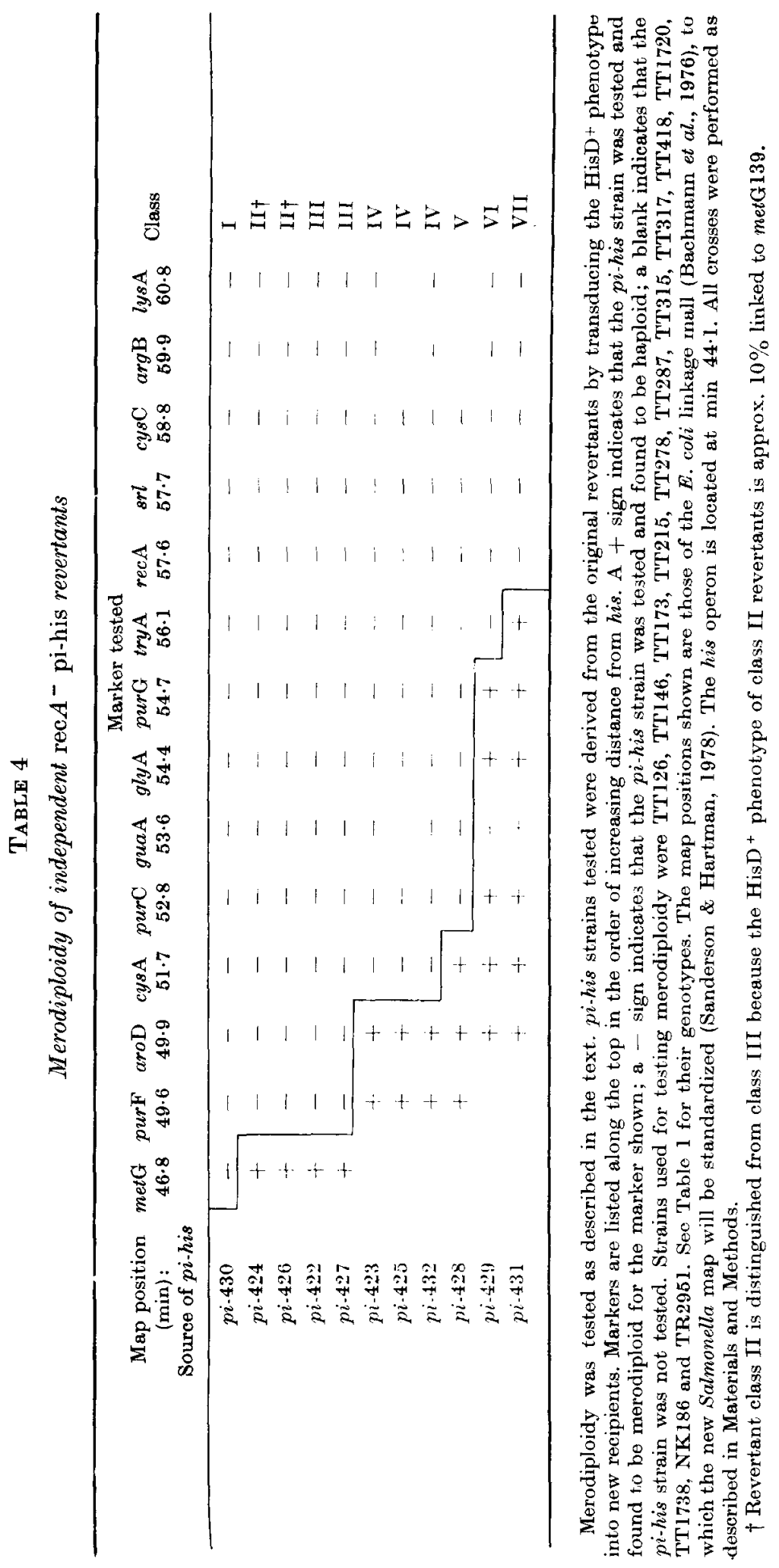


transducing mutant of phage P22 (Schmieger, 1971). The current hisG genetic map includes 80 deletions and 95 point mutations. The deletions define 41 intervals among which the point mutations are distributed.

The procedure for mapping the duplication endpoints within hisG was as follows: each $p i$-his duplication was transduced into deletion mutant his-63, selecting $\mathrm{His}^{+}$. Deletion his-63 removes the entire his $\mathrm{G}$ and his $\mathrm{D}$ genes. Since transduction of the $\mathrm{His}^{+}$phenotype of pi-his revertants involves transducing the join point of at tandem duplication (see above), any hisG or his D sequences carried by his-63 (pi-his) recombinants must be located near the duplication join point. These pi-his strains were used as donors in crosses designed to determine the amount of his material present. Any hisG or his D sequences carried by these strains must have been duplicated in the original pi-his revertant.

The inclusion of hisG material was tested by the ability of his-63 (pi-his) donors to transduce his $\mathrm{G}^{-}$point or deletion mutants selecting $\mathrm{His}^{+}$. The inclusion of his $\mathrm{D}$ sequences was tested by the ability of his-63 (pi-his) donors to transduce his D deletion mutants selecting aminotriazole resistance on minimal medium. When his $\mathrm{D}$ deletion mutants are transduced to $\mathrm{His}^{+}$with pi-his donors, two types of recombinants are possible. The first type is true his ${ }^{+}$recombinants between the recipient deletion and the donor his $\mathrm{D}^{+}$gene. The second type of recombinant arises by reestablishment of the pi-his duplication. Since his $\mathrm{D}^{-}$strains are $h i s \mathrm{G}^{+}$, they can inherit the pi-his merodiploid condition to form complementing $\mathrm{His}^{+}$unstable recombinants (Ames et al., 1963). In testing recombinants for mapping purposes, the second type of recombinant was eliminated by selecting only aminotriazole-resistant clones. The histidine analog 3-amino-1,2,4-triazole is a specific inhibitor of the his $\mathrm{B}$ enzyme (Hilton et al., 1965). Strains are inhibited by aminotriazole if they are unable: to derepress their his $\mathrm{B}$ enzyme levels. True his ${ }^{+}$recombinants are aminotriazoleresistant because they carry a completely normal histidine operon. pi-his merodiploid recombinants are all aminotriazole-sensitive, presumably because the constitutive promoter(s) to which the his $\mathrm{D}$ gene is fused provide insufficient expression of his $\mathrm{B}$ to allow escape from inhibition. The recipient his $\mathrm{B}$ region cannot provide resistance, since only polar his $\mathrm{D}^{-}$deletions were used as recipients: polarity effects prevented high levels of expression of the second his $\mathrm{B}$ copy.

The results of these experiments are shown in Figure 6. Several points merit attention. (1) Each pi-his isolate contains all detectable hisD material. This result is not surprising, since $p i$-his strains are phenotypically His $\mathrm{D}^{+}$. However, the aminoterminal portion of the his $\mathrm{D}$ polypeptide is not required for enzymatic activity. Genetic experiments demonstrate that certain operator-proximal his D deletion mutations retain $h i s \mathrm{D}^{+}$activity (Ino et al., 1975; J. Loper, personal communication). Based on protein sequencing studies, the non-essential region has been estimated to he approximately 80 residues in length (T. Kohno, personal communication). Therefore, it is conceivable that the duplication endpoint might have been located within the his $\mathrm{D}$ gene. (2) Every pi-his isolate obtained in a rec $\mathrm{A}^{+}$background contains no detectable hisG material. All 22 revertants have the same duplication endpoint; this point is located in the region between the his $\mathrm{G}$ and $h i s \mathrm{D}$ genes. Included in these studies was the isolate $p i-2$ described by Ames et al. (1963) and Levinthal \& Yeh (1972). Our mapping results differ from those obtained in the latter study. (3) The location of recA- pi-his endpoints are somewhat heterogeneous. Certain pi-his. duplications formed in rec $\mathrm{A}^{-}$strains have endpoints within the hisG gene. However: 


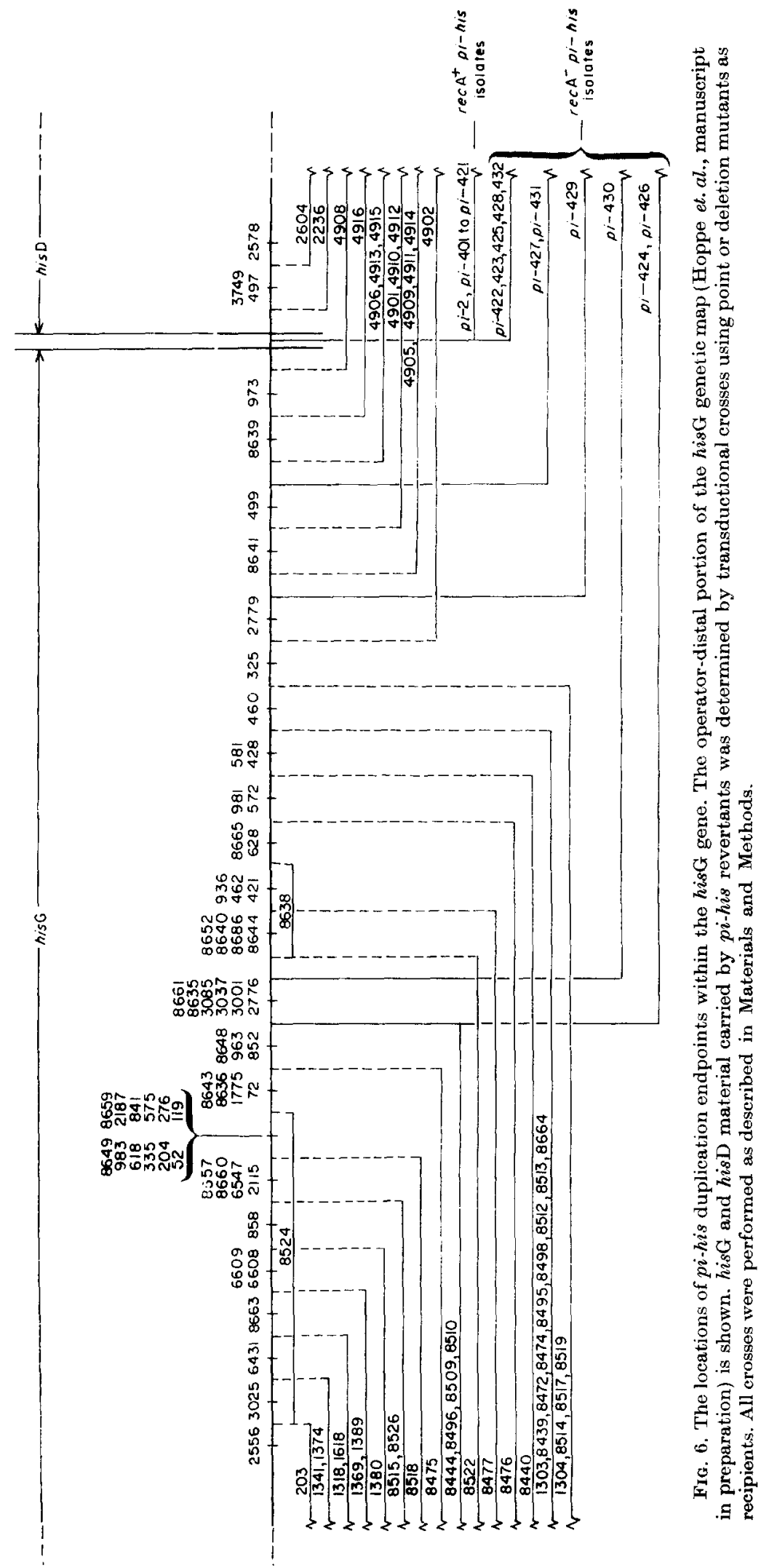


the $h i s \mathrm{G}-h i s \mathrm{D}$ gene boundary again appears to be a preferred site for pi-his duplication formation.

\section{Discussion}

Among the $\mathrm{His}^{+}$revertants of deletion his-203 are strains that are highly unstable for their selected phenotype. In the original description of this phenomenon (Ames et al., 1963) and in more recent work (Levinthal \& Yeh, 1972) the genetic instability was interpreted as evidence that the functional his $\mathrm{D}^{+}$gene in these strains is attached to an extrachromosomal plasmid, termed the pi-his factor. However, numerous instances of genetic instability in both $E$. coli and $S$. Iyphimurium have been attributed to the occurrence of tandem genetic duplications in these organisms (for a review see Anderson \& Roth, 1977a). It seemed to us that pi-his instability might be similarly explained. With this in mind, we have investigated the nature of pi-his revertants. The evidence presented above suggests that pi-his revertants harbor tandem chromosomal duplications which fuse the duplicated his genes to functional promoter elements. This structure (shown in Fig. 4) seems sufficient to account for all properties of $p i$-his revertants.

The experiments designed to detect merodiploidy of nearby genetic markers demonstrate that $p i$-his revertants harbor duplications of genetic material (see Tables 2 and 4). The duplications carried by pi-his revertants have been found to be quite large; individual isolates are duplicated for as much as $25 \%$ of the genome. Tandem duplications of equally large sections of the Salmonella chromosome have heen reported (Straus \& Hoffmann, 1975; Straus \& Straus, 1976 ; Anderson et al., 1976).

As predicted by the nature of tandem duplications, homologous recombination is required for both instability and inheritance of the merodiploid state of pi-his revertants. The stability of $p i$-his revertants in recA- backgrounds (see Fig.5) indicates that pi-his segregation occurs as the result of recombinational events, rather than the partitioning of plasmid molecules among progeny cells. This is strong evidence for a tandem chromosomal location of the duplicated copies. Inheritance of pi-his by transduction also occurs by a recombinational-dependent process (see Fig. 3). When the $\mathrm{His}^{+}$phenotype of pi-his revertants is transduced into rec $\mathrm{A}^{-}$recipients. no recombinants are obtained. These results are consistent with the mechanism for inheritance of tandem duplications outlined in Figure 3 . In contrast, inheritance of E. coli R-factor plasmid molecules by Pl-mediated transduction has been shown to he independent of rec A activity (Ohtsubo, 1970).

Treatment of $p i$-his revertants with agents that stimulate recombination (such as ultraviolet light) increases the rate of His $\mathrm{D}^{-}$segregation (data not shown). The curing of pi-his merodiploidy by acriflavin (Levinthal \& Yeh, 1972) has been interpreted as evidence for plasmids harbored by $p i$-his revertants. However, we have observed that a number of acridine compounds (including acriflavin) efficiently cure known tandem duplications (Anderson \& Roth, manuscript in preparation). We suspect that these compounds increase the amount of recombination, possibly by inducing DNA repair systems (Witkins, 1976). This interpretation is supported by the observation that acriflavin curing of duplications depends on a functional recombination system. Since segregation of tandem duplications results from recombinational processes. increased recombination activity yields increased segregation.

Induced merodiploidy in pi-his recombinants (see Results, section (e)) and cotransduction of the $\mathrm{His}^{+}$unstable phenotype with distant chromosomal markers 
(see Table 3 and Results, section (g)) are perhaps the strongest evidence in support of the tandem duplication model for $p i$-his formation. The requirements for detecting transduction of a tandem duplication (Campbell, 1965; Hill et al., 1969) are most certainly met by the pi-his structure shown in Figure 4 . The functional his $\mathrm{D}^{+}$gene (a selectable marker when used as a donor) is by necessity located near the join point of the tandem duplication, because the join point provides the promoter needed for $h i s \mathrm{D}^{+}$expression. Thus, recipient strains that inherit $p i$-his also inherit the characteristic merodiploidy. Quite often the his $\mathrm{D}^{+}$gene may also be shown to be linked to chromosomal markers unrelated to the $p i$-his selection. These markers are presumably located near the promoters to which the his $\mathrm{D}$ gene has been fused. This linkage is demonstrated for certain revertants by the observation that the three phenotypic properties of the duplication $\left(\mathrm{HisD}^{+}\right.$expression, genetic instability, and chromosomal merodiploidy) can all be simultaneously co-inherited with a particular chromosomal region far from the his region. This non-selective inheritance of a region of merodiploidy is almost certainly the result of cotransducing the join point of a tandem duplication with the particular chromosomal region in question (see Fig. 3).

The most conservative interpretation of these results is that inheritance of pi-his occurs at least initially as a tandem chromosomal duplication. Any chromosome which harbors a tandem duplication can certainly generate covalently closed circular DNA as the result of reciprocal recombination between the two copies of duplicated material. Such molecules have been detected in strains harboring tandem duplications of the $E$. coli gly $\mathrm{T}$ locus (Hill et al, 1977). In many respects, such molecules resemble plasmids. However, they should be lacking the gene(s) or site(s) necessary for autonomous replication. If such molecules can be replicated only by re-inserting into the chromosome, then it seems they may best be considered tandem chromosomal duplications. While we have no direct evidence precluding replication of this molecule, we feel that such replication is unnecessary to account for $p i$-his properties. A search for covalently closed circular DNA in pi-his revertants has been unsuccessful (H. Whitfield, personal communication).

Based on the frequencies and endpoints of pi-his duplications obtained in $r e c \mathrm{~A}^{+}$ and recA- backgrounds, we should like to distinguish between two duplication mechanisms. One mechanism is dependent on recombination function and is responsible for a majority ( 15 of 22 ) of the pi-his revertants obtained in a $r e c \mathrm{~A}^{+}$background. Such revertants ( $r e c A^{+}$class II) are duplicated for the chromosomal region from his through $\arg \mathrm{B}$. The most attractive interpretation of these results is that there exists a DNA sequence in the $\arg \mathrm{B}-l y s \mathrm{~A}$ region which is partially or completely homologous to a sequence found within the his operon at the hisG-his $\mathrm{D}$ border. Legitimate recombination between these sequences yields duplication (or lethal deletion) of intervening material. The remainder of the rec $\mathrm{A}^{+}$revertants (classes $\mathrm{I}$ and III) arise at a frequency comparable to $p$ i-his revertants obtained in a recA- background. Thus, these revertants arise either by recombination-independent processes (see below) or by low frequency, recombination-dependent events.

A second duplication mechanism occurs in the absence of recombination function. It, therefore, satisfies the definition of illegitimate recombination (Franklin, 1971). pi-his duplications generated by this mechanism are heterogeneous with respect to the amount of material duplicated. They occur at a frequency approximately sixfold less than recombination-dependent revertants. Duplications of the $E$. coli argECBH operon have been selected by a similar rationale, and have been reported to occur 
independently of $r e c \mathrm{~A}$ function (Beeftinck et al., 1974). In contrast, duplications of the Salmonella trp operon (selected as revertants of $\operatorname{trp}$ promoter mutations) are not found among revertants in $r e c \mathrm{~A}^{-}$strains; they are frequent among rec $\mathrm{A}^{+}$isolates (Basu \& Margolin, 1972,1973 ; Margolin \& Bauerle, 1966). These results likely reflect differences among strains in sequences available for unequal recombination with the gene under selection. If homologous sequences do not exist (or if they are not located within a functioning transcriptional unit), then only recombination-independent mechanisms are available.

pi-his tandem duplications are rare. They occur spontaneously at a frequency of approximately $3 \times 10^{-10}$ per cell. Other tandem duplications in Salmonella have been estimated to be quite frequent (Miller \& Roth, 1971; Straus \& Hoffmann, 1975; Straus \& Straus, 1976; Anderson et al., 1976; Anderson \& Roth, 1976). Estimates range from $4 \times 10^{-5}$ to $2 \times 10^{-1}$ per cell. Each of these estimates is based on selections which are relatively undemanding in terms of duplication endpoints; the duplications need only include the gene(s) under selection. The pi-his selection, however, is very restrictive of permissible endpoints. One endpoint must be within a small (500 to 1000 bases) region of the his operon; the other must be located within the transcription unit of a functioning and properly oriented promoter. Thus, only a small fraction of total duplications is selected.

The locations of $p i$-his duplication endpoints within the hisG-his $\mathrm{D}$ region are remarkably non-random. Of 33 independent revertants, 27 have endpoints precisely at the his $\mathrm{G}-$-his $\mathrm{D}$ gene boundary. We estimate the target size of permissible endpoints in this region to be 500 to 1000 bases. Endpoints may fall anywhere within the operator-proximal $\sim 240$ base-pairs ( $\sim 80$ amino acids) of the his $\mathrm{D}$ gene, anywhere within the residual hisG gene, or anywhere to the "left" of deletion $h i s-203$ such that no transcription termination sites are encountered (see Fig. 4). Yet most endpoints occur at the his $\mathrm{G}-$ his $\mathrm{D}$ boundary. This curious but unexplained observation may be related to the phenomenon of polarity (Franklin \& Luria, 1961 ; Jacob \& Monod, 1961). Certain duplication events having endpoints within hisG would be expected to generate polar effects at the junction between duplicated regions. If the his D gene is fused to a low-level promoter, these polarity effects might reduce his D expression and prevent utilization of histidinol. Such duplications would not be recovered as pi-his revertants. Duplications with endpoints in the hisG-hisD spacer might be less subject to such polarity effects. Alternatively, the hisG-his D intercistronic spacer might be quite large. However, such a large region has not been revealed genetically (Grabnar el al., 1964).

This work was supported by grants from the United States Public Health Service ((GM-18663) and from the National Science Foundation (PCM76-15048). One author (R. P. A.) was supported by a National Science Foundation predoctoral fellowship.

\section{REFERENCES}

Ahmed, A. (1975). Mol. Gen. Genet. 136, 243-253.

Ames, B. N., Hartman, P. E. \& Jacob, F. (1963). J. Mol. Biol. 7, 23-42.

Anderson, R. P. \& Roth, J. R. (1976). Abstr. Annu. Meeting, Amer. Soc. Microbiol. p. 107, American Society for Microbiology, Washington, D.C.

Anderson, R. P. \& Roth, J. R. (1977a). Annu. Rev. Microbiol. 31, 473-505.

Anderson, R. P. \& Roth, J. R. (1977b). Abstr. Annu. Meeting, Amer. Soc. Microbiol. p. 139. American Society for Microbiology, Washington, D.C.

Anderson, R. P., Miller, C. G. \& Roth, J. R. (1976). J. Mol. Biol. 105, $201-218$. 
Atkins, J. F. \& Loper, J. C. (1970). Proc. Nat. Acad. Sci., U.S.A. 65, 925-932.

Bachmann, B. J., Low, K. B. \& Taylor, A. L. (1976). Bacteriol. Rev. 40, 116-167.

Basu, S. K. \& Margolin, P. (1972). Genetics, 71, Suppl., 3, 3.

Basu, S. K. \& Margolin, P. (1973). Abstr. Annu. Meeting, Amer. Soc. Microbiol. p. 71, American Society for Microbiology, Washington, D.C.

Beeftinck, F., Cunin, R. \& Glansdorff, N. (1974). Mol. Gen. Genet. 132, 241-253.

Bertani, G. \& Bertani, L. E. (1974). Proc. Nat. Acad. Sci., U.S.A. 71, 315-319.

Campbell, A. (1963). Virology, 20, 344-356.

Campbell, A. (1965). Virology, 27, 329-339.

Chattoraj, D. K. \& Inman, R. B. (1974). Proc. Nat. Acad. Sci, U.S.A. 71, $311-314$.

Cunin, R., Elseviers, D. \& Glansdorff, N. (1970). Mol. Gen. Genet. 108, 154-157.

Elseviers, D., Cunin, R. \& Glansdorff, N. (1969). FEBS Letters, 3, 18-20.

Elseviers, D., Cunin, R., Glansdorff, N., Baumberg, S. \& Asheroft, E. (1972). Mol. Gen. Genet. 117, 349-366.

Franklin, N. C. (1971). In The Bacteriophage Lambda (Hershey, A. D., ed.), pp. 175-194, Cold Spring Harbor Laboratory, Cold Spring Harbor.

Franklin, N. \& Luria, S. (1961). Virology, 15, 299-311.

Freedman, R. \& Brenner, S. (1972). J. Mol. Biol. 69, 409-419.

Glansdorff, N. \& Sand, G. (1968). Genetics, 60, 257-268.

Grabnar, M., Stahl, R. C., Hartman, Z. \& Hartman, P. E. (1964). Brookhaven Symp. Biol. $17,15-52$.

Hegeman, G. D. \& Rosenberg, S. L. (1970). Annu. Rev. Microbiol. 24, 429-462.

Hershey, A. D. (1970). Yearb. Carnegie Inst. 69, 717-722.

Hill, C. W. \& Echols, H. (1966). J. Mol. Biol. 19, 38-51.

Hill, C. W., Schiffer, D. \& Berg, P. (1969). J. Bacteriol. 99, 274-278.

Hill, C. W., Grafstrom, R. H., Harnish, B. W. \& Hillman, B. S. (1977). J. Mol. Biol. $116,407-428$.

Hilton, J., Kearney, P. \& Ames, B. N. (1965). Arch. Biochem. Biophys. 112, 544-547.

Ino, I., Hartman, P. E., Hartman, Z. \& Yourno, J. (1975). J. Bacteriol. 123, 1254-1264.

Jackson, E. N. \& Yanofsky, C. (1973). J. Bacteriol. 116, 33-40.

Jacob, F. \& Monod, J. (1961). Cold Spring Harbor Symp. Quant. Biol. 26, 193-211.

Kleckner, N., Chan, R. K., Tye, B. K. \& Botstein, D. (1975). J. Mol. Biol. 97, 561-575.

Levinthal, M. \& Yeh, J. (1972). J. Bacteriol. 109, 993-1000.

Margolin, P. \& Bauerle, R. H. (1966). Cold Spring Harbor Symp. Quant. Biol. 31, 31 1-320.

McEntee, K. (1976). Virology, 70, 221-222.

Miller, C. G. \& Roth, J. R. (I971). J. Mol. Biol. 59, 63-75.

Miller, J. H., Reznikoff, W. S., Silverstone, A. E., Ippen, K., Signer, E. R. \& Beckwith, J. R. (1970). J. Bacteriol. 104, 1273-1279.

Morse, M. L. (1967). Genetics, 56, 331-340.

Ohno, S. (1970). Evolution by Gene Duplication, Springer Verlag, New York.

Ohtsubo, E. (1970). Genetics, 64, 189-197.

Roth, J. R. (1970). Methods Enzymol. 17, 3-35.

Sanderson, K. \& IIartman, I. E. (1978). Bacteriol. Rev. 42, in the pross.

Schmieger, H. (1971). Mol. Gen. Genet. 110, 378-381.

Scott, J. F., Artz, S. W. \& Roth, J. R. (1975). Proc. Nat. Acad. Sci., U.S.A. 72, 5021-5025.

St. Pierre, M. L. (1968). J. Mol. Biol. 38, 71-82.

Straus, D. S. \& Hoffmann, G. R. (1975). Genetics, 80, 227-237.

Straus, D. S. \& Straus, L. D. (1976). J. Mol. Biol. 103, 143-154.

Vogel, H. \& Bonner, D. (1956). J. Biol. Chem. 218, 97-106.

Witkin, E. W. (1976). Bacteriol. Rev. 40, 869-907.

Yudkin, M. D. (1977). J. Bacteriol. 130, 57-61. 